\title{
Avanços e desafios do acolhimento na operacionalização e qualificação do Sistema Único de Saúde na Atenção Primária: um resgate da produção bibliográfica do Brasil
}

\author{
Progress and challenges facing user acceptance \\ in the implementation and qualification of the Unified Health \\ System in Primary Healthcare: a review \\ of the bibliographical output in Brazil
}

Sandra Minardi Mitre ${ }^{1}$

Eli Iola Gurgel Andrade ${ }^{2}$

Rosângela Minardi Mitre Cotta ${ }^{3}$

${ }^{1}$ Programa de Pós-Graduação em Saúde Pública, Faculdade de Medicina, Universidade Federal de de Minas Gerais. Av. Prof. Alfredo Balena 190, Santa Efigênia. 30130100 Belo Horizonte MG. sandraminardi@hotmail.com ${ }^{2}$ Departamento de Medicina Preventiva e Social, Faculdade de Medicina da Universidade Federal de Minas Gerais.

${ }^{3}$ Programa de Inovação em Docência Universitária da Área da Saúde, Laboratório de Estudos em Planejamento e Gestão em Saúde, Departamento de Nutrição e Saúde, Universidade Federal de Viçosa, Minas Gerais.
Abstract The public policies adopted by the Unified Health System (SUS) in Brazil have gone through successive transformations, striving to reassert health as a universal right. The user acceptance of the guidelines of the National Humanization Policy for Care and Management of the SUS Humanize SUS - is taking shape and relevance in Primary Healthcare (PHC) to ensure humanized access and resolution of the health demands of users and communities in Brazil. A critical analysis of the bibliographical output in Brazil from 1989 to 2009 was conducted regarding acceptance of implementation and qualification of SUS in PHC. The databases consulted were SciELO, Lilacs and Medline. The results revealed progress in broadening access to PHC services and health professionals more sensitive to the needs of users and communities. However, lack of coordination in integrated networks, excess demand, the hegemonic biomedical model, lack of training and democratic and reflexive spaces to reorganize the work process have been raising increasingly more incisive questions about the potential of this guideline for the implementation and qualification of SUS.

Key words User acceptance, Primary Healthcare, Family Health Program, Unified Health System, Health policy, Health services evaluation
Resumo As políticas públicas adotadas pelo Sistema Único de Saúde (SUS) têm passado por sucessivas transformações, buscando reafirmar a saúde como direito universal. $O$ acolhimento, diretriz operacional da Política Nacional de Humanização da Atenção e Gestão do SUS (Humaniza SUS), vem ganhando contornos próprios e relevância na atenção primária à saúde (APS) para garantir acesso humanizado e resolubilidade às demandas de saúde dos usuários e das comunidades no Brasil. Realizou-se uma análise crítica da produção bibliográfica, no Brasil, no período de 1989 a 2009, sobre o acolhimento na operacionalização e qualificação do SUS na APS. As bases de dados consultadas foram SciELO, Lilacs e Medline. Os resultados apontaram avanços na ampliação do acesso aos serviços da APS e profissionais de saúde mais sensíveis às necessidades dos usuários e comunidades. A ausência de articulação em redes integradas, o excesso de demanda, o modelo biomédico hegemônico, a ausência de capacitação e de espaços democráticos e reflexivos para reorganizar o processo de trabalho em saúde têm colocado em questão, de modo cada vez mais incisivo, a potencialidade desta diretriz na operacionalização e qualificação do SUS.

Palavras-chave Acolhimento, Atenção Primária à Saúde, Programa de Saúde da Família, Sistema Único de Saúde, Política de saúde, Avaliação de serviços de saúde 


\section{Introdução}

O direito à saúde foi reconhecido internacionalmente em 1948, quando da aprovação da Declaração Universal dos Direitos Humanos pela Organização das Nações Unidas. Na Conferência Internacional sobre cuidados primários à saúde, realizada em 1978, a Declaração de Alma-Ata reafirmou a saúde como um direito humano universal ${ }^{1}$. No Brasil, na década de 70, a Reforma Sanitária buscou garantir a saúde como direito individual, traduzida na equidade e no acesso universal a um sistema público de saúde, solidário e inclusivo ${ }^{2,3}$. A VIII Conferência Nacional de Saúde, em 1986, buscou transformar o arcabouço jurídico-institucional do setor saúde e assegurá-la como direito de todo cidadão brasileiro ${ }^{4}$. Dois anos depois, a Constituição da República anunciou as garantias para sistematizar as ações e os serviços de saúde inscritos pela universalidade do acesso, equidade e integralidade da assistência em um Sistema Único de Saúde (SUS) ${ }^{2,3}$. De acordo com Nascimento e Zioni ${ }^{5}$, o SUS manifesta-se como um movimento de resistência à exclusão, em defesa dos direitos universais.

No início da década de 90, a tendência nacional para enfrentar as iniquidades sociais e ampliar o acesso aos serviços de saúde, tendo na atenção primaria à saúde (APS) a porta de entrada ao sistema sanitário, começava a ser modificada por iniciativa de alguns municípios, que implantaram o Programa de Agentes Comunitários (PACS). Ao ampliar e reordenar as ações da rede primária, em 1994, o Ministério de Saúde implantou o Programa de Saúde da Família (PSF), aspirando superar o modelo centrado na doença e tendo como alvo as demandas individuais e coletivas para o cuidado integral e continuado ${ }^{6}$. Quando implantado, o PSF estava limitado aos grupos populacionais de maior risco social e expostos às precárias condições sanitárias, o que ocorreu predominantemente em municípios de pequeno porte, enquanto os de maior população apresentaram pouca adesão $0^{6,7}$.

A despeito destas questões, o SUS, ao longo da década de 90, foi considerado excludente pelos significativos obstáculos impostos para a sua efetivação ${ }^{8,9}$. O acesso aos serviços públicos era difícil, suas instalações e equipamentos eram insuficientes e sucateados, marcados por enormes filas e pelo baixo nível de qualidade e resolubilidade, herança do modelo previdenciário ${ }^{7,10}$. Além disto, as práticas dos profissionais de saúde eram tidas como impessoais e fragmentadas. A imagem da assistência pública era negativa, com o apoio da mídia que enfatizava a eficiência e a qualidade do setor privado em detrimento ao público ${ }^{7,10,11}$.

Ademais, de acordo com Cecílio ${ }^{12}$, a rede de APS não conseguia tornar-se a porta de entrada do sistema, que continuava a ser o hospital. Os pronto-socorros estavam sempre lotados, com a evidência de que o perfil de morbidade da clientela atendida nestas unidades poderia ter resolubilidade ao nível primário. Segundo Merhy ${ }^{13,14}$, associado a este quadro estava o baixo impacto que as ações da APS tinham sobre os principais problemas de saúde da população, evidenciado pelo aumento da incidência de doenças evitáveis e erradicáveis, apesar dos avanços científicos e tecnológicos já alcançados.

Tornou-se imperativo reconstruir o modo de produzir e de operar as ações de saúde no SUS, que se comprometesse com a defesa da vida e com os direitos sociais plenos, e, ao mesmo tempo, pudesse dar resolubilidade aos problemas identificados no dia a dia do trabalho, orientados para a autonomia dos usuários e das comunidades ${ }^{13,14}$. Surge então, em meados da década de 90, o acolhimento, que ganha o discurso de inclusão social em defesa do SUS, um dispositivo capaz de disparar reflexões e mudanças na organização dos serviços de saúde, na retomada do acesso universal, no resgate da equipe multiprofissional e na qualificação das relações entre usuários e profissionais de saúde ${ }^{13,15,16}$.

A partir de 2000, o debate em torno do acolhimento se intensifica na perspectiva da humanização da saúde, anunciada pela XI Conferência Nacional de Saúde (CNS), que apontava para as dificuldades enfrentadas pelo SUS e sua incompatibilidade com o modelo econômico vigente, caracterizado pelo autoritarismo, clientelismo, exclusão e dificuldade de organização social com $\mathrm{o}$ aumento das iniquidades sociais ${ }^{17}$. A proposta de "Reforma do Estado" contemplava a cessão dos serviços públicos "lucrativos ao setor privado; a concentração da ação direta do Estado em programas de baixo custo e voltados às populações pobres, e a ampliação dos recursos dos planos de saúde para camadas medias da população"18. Neste contexto, nova crise se instaura, assinalada pela dificuldade do acesso aos serviços do SUS e pela baixa qualidade da assistência prestada. De acordo com Benevides e Passos ${ }^{17}$, de um lado estavam os usuários, que buscavam a atenção com acolhimento e de modo resoluto, e de outro os profissionais de saúde, que reivindicavam melhores condições de trabalho com acesso à capacitação profissional e às relações democráticas com os gestores. 
Já em 2003, em clima democrático renovado com vistas às novas políticas sociais e econômicas que assegurassem o desenvolvimento econômico sustentável do país, distribuição de renda e inclusão social, foi realizada a XII CNS, que retoma o debate em torno da universalidade do acesso ao SUS, da valorização dos usuários e dos trabalhadores na participação e na gestão do sistema ${ }^{19}$. Surge então, em 2003, a Política Nacional de Humanização da Atenção e Gestão do SUS Humaniza SUS (PNH) ${ }^{17,20}$, que veio para afirmar a indissociabilidade entre a atenção e a gestão dos processos de produção de saúde, assegurar a inclusão de usuários e trabalhadores na gestão dos serviços de saúde, e impulsionar ações para disparar processos no plano das políticas públicas para transformar os modelos de atenção e da gestão da saúde.

Neste novo cenário, o acolhimento ganha o discurso oficial do Ministério da Saúde ${ }^{21}$ se configurando como uma das diretrizes de maior relevância da PNH para operacionalização do SUS; que propõe o protagonismo de todos os sujeitos envolvidos no processo de produção de saúde, a reorganização dos serviços a partir da problematização dos processos de trabalho, além de mudanças estruturais na forma de gestão para ampliar os espaços democráticos de discussão, de escuta e de decisões coletivas.

Sem embargo, a temática do acolhimento vem ganhando contornos próprios e uma importância crescente para o SUS ${ }^{22}$, enquanto a APS cresce em relevância para a reorientação do modelo assistencial, evidenciado pela expansão do PSF nos grandes centros urbanos, além dos municípios de pequeno e médio porte ${ }^{6}$. Desde esta perspectiva, o objetivo do presente estudo foi realizar uma análise crítica-reflexiva da produção bibliográfica do Brasil, dos últimos vinte anos, acerca do acolhimento e de suas implicações na operacionalização e qualificação do SUS na APS.

\section{Método}

Este estudo de natureza bibliográfica foi realizado por meio de uma revisão da literatura, centrada no período de 1989-2009. As informações sobre o material bibliográfico concentraram-se nas publicações da Biblioteca Virtual em Saúde (BVS), nas bases de dados Lilacs (Literatura Latino-Americana em Ciências da Saúde), Medline (Literatura Internacional em Ciências da Saúde) e SciELO (Scientific Electronic Library Online). Para a consulta nestas bases de dados utilizou-se o descritor Acolhimento, conjugado com Atenção Primária à Saúde e Programa de Saúde da Família.

Na busca com a utilização destes descritores em "assuntos" encontrou-se 14 referências. Contudo, observou-se que tais produções aparecem somente a partir de 2004. Diante da necessidade de conhecer estudos realizados no Brasil, no período de janeiro de 1989 a dezembro de 2009, ampliou-se a busca para "título", expandindo o número de referências para um total de 36 de publicações. Dados os objetivos do estudo, foram excluídas as teses, monografias, resenhas, editoriais, ensaios e aqueles que se repetiam, resultando na seleção de 28 artigos.

Os artigos selecionados foram organizados por ano de publicação, localidades, sujeitos participantes e pelo delineamento dos estudos. Em seguida, seguindo os preceitos metodológicos de $\operatorname{Bardin}^{23}$, os núcleos de conteúdo que compõem esta revisão foram sistematizados em mais dois recortes: o primeiro analisou a evolução conceitual e as dimensões do acolhimento utilizadas nas publicações; enquanto o segundo analisou os temas abordados: o acesso, as redes integradas, os modelos assistenciais, os protocolos ou critérios de risco, a educação permanente, a organização do trabalho, a ambiência e tecnologia informacional, categorizados como avanços e desafios para a operacionalização e qualificação do SUS.

\section{Resultados e Discussão}

\section{Um panorama geral no Brasil do acolhimento na atenção primária à saúde}

As primeiras publicações sobre a temática do acolhimento na atenção básica foram realizadas a partir 1999, com experiências pioneiras nos municípios de Betim (MG) ${ }^{15}$ e Belo Horizonte $(\mathrm{MG})^{24}$; enquanto em 2000, surgem publicações em outros municípios, como Ribeirão Preto $(\mathrm{SP})^{25}$ e Curitiba $(\mathrm{PR})^{26}$. Como pode ser observado no Gráfico 1, houve um incremento das publicações em 2004, que segundo Benevides e Passos $^{27}$, foi o ano de criação do Prêmio David Capistrano, que incentivou a publicação de iniciativas e experiências inovadoras para a construção de um SUS humanizado e que contou com a participação de usuários, profissionais e gestores. No entanto, no ano posterior houve um declínio de publicações, com ascensão a partir de 2006. 
Em relação às regiões onde as pesquisas sobre o acolhimento foram realizadas, pode-se observar na Tabela 1 que as publicações concentraram-se na Região Sudeste (46\%), seguidas pelas Regiões Sul (29\%) e Nordeste (26\%). Entende-se que qualquer conhecimento científico

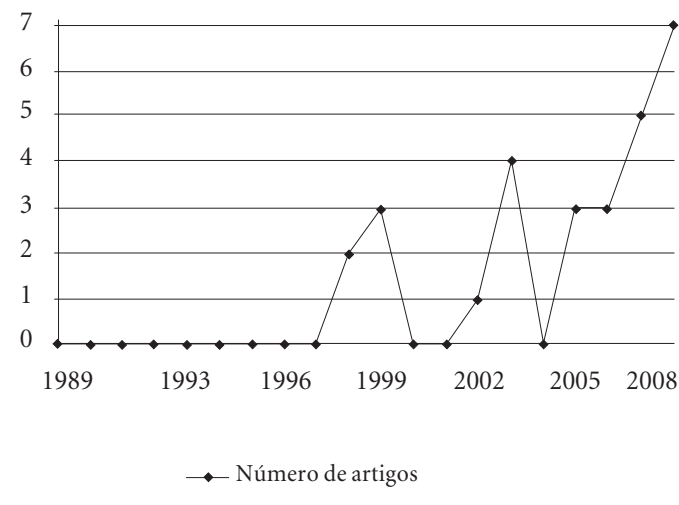

Gráfico 1. Distribuição dos artigos selecionados por ano de publicação, no período de 1989 a 2009

Fonte: Bases de dados LILACS, MEDLINE e SciELO, no período de 1989 a 2009

Tabela 1. Distribuição dos artigos analisados, segundo localização, Brasil

\begin{tabular}{lrr}
\hline REGIÃO - Município / Estado & n & \% \\
\hline SUDESTE & $\mathbf{1 3}$ & $\mathbf{4 6}$ \\
Betim - MG & 1 & 4 \\
Belo Horizonte - MG & 3 & 11 \\
Ribeirão Preto - SP & 3 & 8 \\
São Paulo - SP & 3 & 11 \\
Campinas - SP & 1 & 4 \\
São Carlos - SP & 1 & 4 \\
Santana do Parnaíba - SP & 1 & 4 \\
SUL & $\mathbf{8}$ & $\mathbf{2 9}$ \\
Curitiba - RS & 2 & 7 \\
Porto Alegre - RS & 2 & 7 \\
Balneário de Comburiu - SC & 1 & 4 \\
Londrina - PR & 2 & 7 \\
Não Especificado - RS & 1 & 4 \\
NORDESTE & 7 & $\mathbf{2 6}$ \\
Fortaleza - CE & 3 & 11 \\
Campina Grande - PB & 1 & 4 \\
Alagoinha - BA & 1 & 4 \\
Aracaju - SE & 1 & 4 \\
Não Especificado - RN & 1 & 4 \\
Total & $\mathbf{2 8}$ & $\mathbf{1 0 0}$ \\
\hline
\end{tabular}

Fonte: Bases de dados Lilacs, Medline e SciELO, no período de 1989 a 2009 produzido busca a articulação entre a teoria e a realidade empírica, portanto há de se preocupar com a ausência de publicações nas Regiões Centro-Oeste e Norte do Brasil, quando se discute o acolhimento como possibilidade de inclusão social, em defesa do SUS humanizado ${ }^{15,22}$ e "que dá certo" 27 .

Pode-se constatar pelo Gráfico 2, que a maioria dos estudos teve abordagem qualitativa (89\%). Na Tabela 2, observa-se que os profissionais e usuários foram seus maiores protagonistas. Vale destacar que, segundo Minayo ${ }^{28}$, historicamente, no campo da saúde houve uma ten-

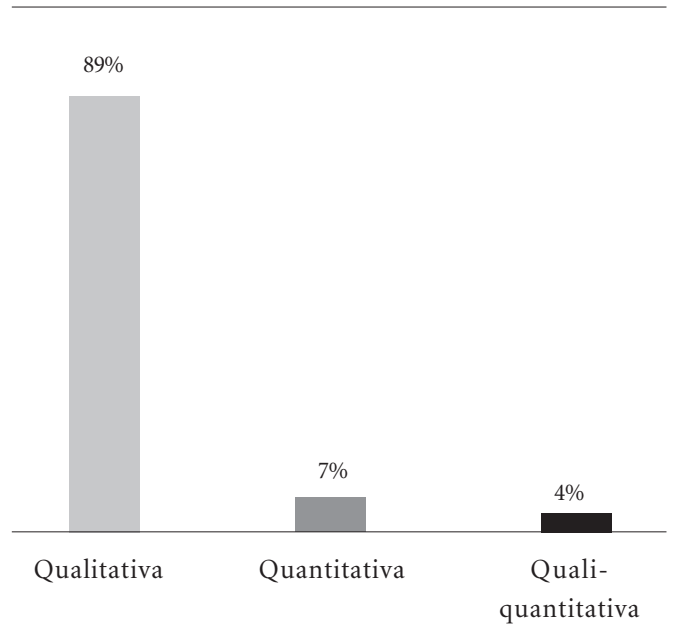

Gráfico 2. Distribuição dos artigos analisados, segundo o delineamento do estudo

Fonte: Bases de dados LILACS, MEDLINE e SciELO, no período de 1989 a 2009

Tabela 2. Distribuição dos artigos analisados, segundo os sujeitos pesquisados

\begin{tabular}{lrr}
\hline \multicolumn{1}{c}{ Sujeitos } & n & \% \\
\hline Profissionais de saúde & 12 & 43 \\
Usuários & 5 & 18 \\
Profissionais e usuários & 5 & 18 \\
Profissionais e gestores & 2 & 7 \\
Profissionais e Conselheiros & 1 & 3 \\
Profissionais, usuários e estudantes do PSF & 1 & 3 \\
Não se aplica ( organização do serviço) & 2 & 7 \\
Total & $\mathbf{2 8}$ & $\mathbf{1 0 0}$
\end{tabular}

Fonte: Bases de dados Lilacs, Medline e SciELO, no período de 1989 a 2009 
dência de transformar sujeitos em doentes/pacientes, enquanto na organização dos serviços, em relação ao planejamento e à avaliação destacavam-se os métodos que conferiam relevância às relações entre cargos, papéis e técnicas. Mesmo no campo organizacional do planejamento estratégico, a ênfase ainda volta-se para a observação dos diferentes atores, buscando controlá-los, ao invés de aclamar por sua participação. Em contrapartida, os estudos analisados sobre o acolhimento buscaram alcançar o sujeito na qualificação do cuidado em saúde e na gestão do processo de trabalho. Assim, ao trazer a reflexão sobre estes, possibilitou-se reconhecê-los como seres autênticos, dotados de necessidades e de valores próprios, capazes de produzir história e corresponsabilizaçãa ${ }^{29,30}$.

Ademais, os estudos analisados expressaram como esta diretriz foi absorvida nas práticas do cotidiano dos serviços de saúde, ao reunir diferentes perspectivas e interesses de vários sujeitos: profissionais de saúde, usuários, conselheiros e gestores do SUS.

\section{Acolhimento: múltiplos conceitos e dimensões}

Uma das publicações pioneiras sobre o acolhimento na APS foi realizada por Franco et al. ${ }^{15}$, que propõem discuti-lo como uma diretriz operacional, pautada nos princípios do SUS para atender a todas as pessoas que procuram os serviços, garantindo a universalidade no acesso; buscar a reorganização do processo de trabalho, deslocando o eixo centrado no médico para uma equipe multiprofissional, capaz de produzir a escuta qualificada, responsabilização, vínculo e resolubilidade; além de qualificar a relação dos profissionais com os usuários, sob parâmetros humanitários, de solidariedade e cidadania. Os autores destacam que o acolhimento deve alcançar a dimensão da gestão do processo de trabalho, pois ele só será "possível se a gestão for participativa, baseada em princípios democráticos e de interação entre a equipe, [...], pois a mudança no processo de trabalho pressupõe a adesão dos trabalhadores à nova diretriz"15.

Em publicações posteriores o acolhimento aparece como uma ferramenta ${ }^{24}$, estratégia ${ }^{31}$, e arranjo tecnológico ${ }^{32}$ utilizado para iniciar mudanças no processo de trabalho em saúde, garantir o acesso em serviços humanizados, resolutos e de qualidade com a responsabilização coletiva dos trabalhadores às necessidades dos usuários. Nestes estudos, no entanto, a dimen- são do acolhimento em torno de uma gestão participativa não é debatida, e a resolubilidade pelos problemas de saúde do usuário passa a ser discutida como responsabilização dos profissionais de saúde. Nos resultados destes estudos, observa-se a dificuldade de compreensão, pelos profissionais de saúde, do processo de trabalho no acolhimento, que o reconhecem como uma espécie de "triagem humanizada" e já apontam para a sobrecarga de trabalho nas UAPS (Unidades de Atenção Primária à Saúde).

Em outra publicação ${ }^{33}$, o acolhimento aparece como um pilar fundamental para a construção do novo modelo de APS, definido por critérios técnicos, éticos e humanos, no qual os profissionais devem receber a demanda, buscar formas de resolubilidade, mas que não resultará, necessariamente, na resolução completa dos problemas referidos pelo usuário. Ao profissional, cabe dispensar a atenção ao usuário, o que envolve escuta, valorização da queixa e identificação das necessidades individuais e coletivas. No resultado deste estudo, os profissionais de saúde associaram o acolhimento à sala de espera, praticado como mais uma tarefa, dentre tantas executadas na UAPS.

Nesta direção, Cunha ${ }^{34}$ alerta que à medida que o profissional de saúde incorpora uma ferramenta ou atividade como uma rotina, neste caso o acolhimento, acaba por esquecer ou não reconhecer sua motivação e seus fundamentos conceituais, passando a executá-lo de forma acrítica. Esta alienação pode ter consequências para a saúde dos trabalhadores e para a eficácia do cuidado nos serviços de saúde. De acordo com Tesser et al..$^{35}$, quanto menos ritualizadas as ações, mais flexíveis os profissionais e trabalhando juntos, maior será a possibilidade de troca de saberes para realizar o acolhimento. Isto requer a "comunicação, interpretação e negociação permanente entre a equipe e com os usuários, estimulando o vínculo, acalmando ansiedades e buscando soluções".

Em estudo desenvolvido por Takemoto e Sil$\mathrm{va}^{36}$ amplia-se o debate em torno do acolhimento como uma postura diante do usuário em todos os momentos, além de um dispositivo capaz de reorganizar o processo de trabalho na UAPS. No entanto, como resultados os autores alertam para a atribuição exclusiva do acolhimento a um grupo de recepção, o que gera sobrecarga de trabalho e pouca adesão dos demais profissionais de saúde a esta diretriz. Em conclusão, resgatam a necessidade do apoio da gestão para a produção de espaços de reflexão e capacitação do profissio- 
nal, retomando a discussão da dimensão do acolhimento inserida em uma gestão participativa.

Em estudos que propõem pesquisar a percepção do usuário, o acolhimento é discutido como uma postura dos profissionais na recepção, considerada positiva quando baseada no respeito, consideração e atenção dispensada aos usuários do serviço ${ }^{37}$. O acolhimento aparece também referenciado no modelo de ajuda, que requer dos profissionais habilidades pessoais para se interessar, compreender e responsabilizar-se pelos problemas do usuário ${ }^{25}$. Estas publicações, porém, não apontam para o processo de trabalho do acolhimento, e nem para a dimensão da gestão do trabalho em saúde.

Vale destacar que a identificação dos problemas de saúde trazidos pelo usuário, integrando à compreensão do seu contexto de vida e dos recursos mobilizados por ele, diante de tantas iniquidades, requer a inclusão pelos profissionais da "arte da alteridade" como ponto chave na produção do cuidado ${ }^{38}$. Esta, congrega a noção de humanidade plural, que ao negar a existência de um centro do saber, aceita a diversidade de culturas e é capaz de apreender os costumes alheios a partir da diferença ${ }^{39}$. Deste modo, reconhece-se, que o usuário detém o poder sobre suas escolhas, portanto, deve ser o sujeito na definição de finalidades e dos meios do trabalho em saúde. Para Ayres ${ }^{40}$, esta perspectiva constitui uma nova interface dialógica, que terá um enorme potencial para a reconstrução das práticas de saúde. Assim, a corresponsabilização assume importância fundamental no cuidado, que engloba a construção do vínculo serviço-usuário, a garantia do controle social das políticas públicas e a gestão dos serviços de saúde.

Ademais, vale lembrar que o acolhimento proposto pela $\mathrm{PNH}^{41}$ ancora-se no protagonismo dos sujeitos envolvidos no processo de produção de saúde, que incluem os usuários, os profissionais de saúde e os gestores, enfatizando a necessidade de reorganizar o serviço de saúde com mudanças na forma de gestão, a partir da ampliação dos espaços interdisciplinares e democráticos de discussão.

Recentemente, o conceito de "acolhimento coletivo" na APS foi introduzido por Cavalcante Filho et al. ${ }^{42}$ como um dispositivo para ampliar a acessibilidade aos serviços de saúde, estruturar o processo de trabalho centrado nas necessidades da população, com potencial para formar novas maneiras de produzir o cuidado. Um espaço capaz de incluir a voz do usuário na construção de projetos terapêuticos, e de integrar os profissionais e seus saberes numa perspectiva "interdisciplinar", na qual os saberes e tecnologias circulam em benefício do cuidado. Para estes autores, o acolhimento coletivo não exclui a necessidade do acolhimento individual e enfatizam um processo de trabalho que resulte, ao mesmo tempo, na realização profissional dos membros da equipe.

Diante do exposto, faz-se necessário identificar e discutir as linhas de força, os avanços, os pontos críticos e os desafios do acolhimento na operacionalização e na qualificação do SUS na APS (Quadro 1).

\section{Avanços e desafios do acolhimento para o Sistema Único de Saúde na atenção primária}

A partir da implantação do acolhimento, a ampliação do acesso aos serviços de APS foi evidenciada nos estudos analisados ${ }^{15,24,26,31,36,37,43-48}$, como apresentado no Quadro 1. Contudo, levantam-se questões importantes sobre a definição de acesso. Para Donabedian ${ }^{49}$ seria a capacidade do usuário do serviço de saúde obter, quando necessita, cuidado à sua saúde de maneira fácil e conveniente; já de acordo com Travasso e Martins ${ }^{50}$, quando relacionado ao serviço, o acesso está associado ao desempenho e à oferta de cuidados que podem ser oferecidos, enquanto que para o usuário o uso efetivo do serviço resultaria de uma multiplicidade de fatores, como individuais predisponentes, fatores contextuais e aqueles relativos à qualidade do cuidado ofertado. Assim, a medida do acesso é complexa, e no caso dos estudos analisados nesta revisão foram utilizadas medidas indiretas que, segundo Malta e Merhy ${ }^{51}$, indicaram o aumento da oferta dos serviços pelo aumento da capacidade instalada representada na ampliação do número de atendimentos realizados e da demanda direcionada aos profissionais de saúde.

A territorialização foi um fator facilitador do acesso dos usuários à ABS, citado em alguns estudos ${ }^{15,24,37}$, o que consiste em um conceito técnico na definição de territórios para distinção de responsabilidades sanitárias ${ }^{52}$. Nestes estudos, observou-se que os municípios se dividiram em territórios com margens de responsabilização sanitária para implementar as áreas de abrangência, o que facilitou as ações em saúde pelas equipes do PSF.

O primeiro e grande desafio constatado e apresentado no Quadro 1 foi a dificuldade de acesso aos exames e às consultas especializadas, e 
Quadro 1. Caracterização da temática abordada nos estudos sobre o Acolhimento analisados e os principais resultados categorizados como avanços e desafios

\begin{tabular}{|c|c|}
\hline Temática & Resultados \\
\hline Acesso & $\begin{array}{l}\text { Avanços } \\
\text { - Garante a ampliação do acesso nas Unidades de Atenção primária à saúde (UAPS) } \\
\text { pelos usuários do SUS } S^{15,24,26,31,36,37,43-48} \text {. } \\
\text { - A organização territorial das UAPS facilita o acesso }{ }^{15,24,37} \text {. } \\
\text { Desafios } \\
\text { - Persistem dificuldades no acesso aos exames e as consultas especializadas }{ }^{15,31,43,45,46,53-56} \text {. }\end{array}$ \\
\hline $\begin{array}{l}\text { Redes } \\
\text { Integradas }\end{array}$ & $\begin{array}{l}\text { Desafios } \\
\text { - Ausência de mecanismos de comunicação e integração da APS com demais níveis de } \\
\text { complexidade do SUS. Fragilidade no mecanismo de Referência e Contrarreferência }{ }^{15,24,31} \text {, } \\
\text { 53-55,57,58. } \\
\text { - Ausência de prontuários unificados e com informações gerais do usuário para serem } \\
\text { referendados a outros níveis de atenção, dificulta a comunicação e a realização do } \\
\text { projeto terapêutico integrado e centrado no usuário }{ }^{43} \text {. }\end{array}$ \\
\hline $\begin{array}{l}\text { Modelo } \\
\text { Assistencial }\end{array}$ & $\begin{array}{l}\text { Avanços } \\
\text { - Profissionais mais sensíveis as demandas do usuário }{ }^{25,33,37,43,59} \text {. } \\
\text { Desafios } \\
\text { - A manutenção da hegemonia no trabalho em saúde centrado no modelo clínico e na } \\
\text { consulta médica dificulta a autonomização do usuário }{ }^{24,36,49} \text {. } \\
\text { - A atenção centrada na queixa e que define a conduta (queixa-conduta) faz com que o } \\
\text { usuário tenha que exagerar na queixa para ser atendido }{ }^{55} \text {. } \\
\text { - O modelo biomédico, centrado na doença, enfatizado pelos profissionais e demandados } \\
\text { pelos usuários deixa pouco espaço para ações educativas, de promoção e prevenção à } \\
\text { saúde }{ }^{15,24,32,57} \text {. } \\
\text { - Pouca valorização dos saberes, crenças e habilidades dos usuários pelos profissionais de } \\
\text { saúde, associado ao despreparo para lidar com a subjetividade deste levam a tendência a } \\
\text { culpabilizá-lo por seus problemas de saúde }{ }^{42,43,57,60,61} \text {. } \\
\text { - Necessidade de outros profissionais na UBS como fisioterapeutas, nutricionistas, } \\
\text { terapeutas ocupacionais, dentre outros para o trabalho interdisciplinar }{ }^{44} \text {. }\end{array}$ \\
\hline $\begin{array}{l}\text { Protocolos - } \\
\text { Critérios de } \\
\text { Riscos }\end{array}$ & $\begin{array}{l}\text { Avanços } \\
\text { - O uso de protocolos - critérios de prioritários - garante a priorização do atendimento a } \\
\text { partir da identificação de riscos e necessidades, e não apenas por critérios burocráticos e } \\
\text { administrativos } \text { s }^{15,33,36,45,47,61} \text {. } \\
\text { - O uso do protocolo associado à capacitação e ao trabalho em equipe é mais eficiente na } \\
\text { atenção centrada no usuário }{ }^{61} \text {. } \\
\text { - A sensibilização, a participação na elaboração e a compreensão dos profissionais de } \\
\text { saúde sobre os protocolos facilitam o seu uso na direção da clinica ampliada }{ }^{15,24,31,47,39} \text {. } \\
\text { - A flexibilização no uso dos protocolos permite ampliar e qualificar a escuta }{ }^{42} \text {. } \\
\text { Desafios } \\
\text { - A rigidez na utilização dos protocolos leva ao risco de manter a avaliação do profissional de } \\
\text { saúde somente para a queixa-conduta e aos aspectos clínicos dos problemas de saúde } \\
\text { apresentados pelo usuário, limitando a clinica ampliada }{ }^{49,51,60} \text {. } \\
\text { - O atendimento do usuário somente centrado pela gravidade do problema de saúde faz com } \\
\text { que ele tenha que exagerar na queixa para ser atendido }{ }^{49,51,60} \text {. } \\
\text { - A percepção do usuário de que o profissional é quem decide por ele - se deve ou não ser } \\
\text { atendido pelo médico - gera insatisfação e limitação de sua autonomia }{ }^{49,51,60} \text {. }\end{array}$ \\
\hline $\begin{array}{c}\text { Educação } \\
\text { Permanente }\end{array}$ & $\begin{array}{l}\text { Desafios } \\
\text { - Poucos mecanismos de acesso para a capacitação dos profissionais de saúde } 24,31-33 \text {, } \\
44,55,57,62 \text {. }\end{array}$ \\
\hline
\end{tabular}


Quadro 1. continuação

\begin{tabular}{|c|c|}
\hline Temática & Resultados \\
\hline $\begin{array}{l}\text { Organização } \\
\text { do trabalho }\end{array}$ & $\begin{array}{l}\text { Desafios } \\
\text { - Pouca adesão e mudança no processo de trabalho dos médicos }{ }^{15,31,48,55,62} \text {. } \\
\text { - A atribuição do acolhimento para somente um grupo de profissionais, apesar de propiciar } \\
\text { maior agilidade, pode contribuir para pouca adesão e o descompromisso dos demais } \\
\text { profissionais da equipe pela mudança no processo de trabalho }{ }^{36,63} \text {. } \\
\text { - O excesso de demanda e a falta de profissionais de saúde geram a sobrecarga de trabalho, } \\
\text { cansaço e estresse nos trabalhadores }{ }^{24,31-33,55,62} \text {. } \\
\text { - A pressão pelo atendimento rápido causa sofrimento nos profissionais de saúde }{ }^{44,46} \text {. } \\
\text { - O medo da agressividade de alguns usuários, quando não há mais vagas para as } \\
\text { consultas, gera estresse nos trabalhadores } \\
\text { - Existência de práticas conflitivas entre normatização administrativa e demandas dos } \\
\text { usuários }{ }^{51} \text { - Exigências dos gestores para a realização de tarefas administrativas em } \\
\text { detrimento das atividades assistenciais }{ }^{32} \text {. } \\
\text { - Fragmentação do processo de trabalho e ausência de participação dos profissionais nas } \\
\text { resoluções e deliberações da unidade }{ }^{43} \text {. } \\
\text { - Conflitos e desarticulação das equipes. Ausência de reuniões e espaços para discussão e } \\
\text { reflexões das equipes. As reuniões são apenas informativas e normativas }{ }^{31,36,44,49,55,63} \text {. } \\
\text { - Precárias condições de trabalho dos Agentes comunitários de saúde - ACS }{ }^{46,48} \text {. } \\
\text { - Pouca valorização do usuário pelo trabalho do auxiliar de enfermagem }{ }^{44} \text {. }\end{array}$ \\
\hline Ambiência & $\begin{array}{l}\text { Avanços } \\
\text { - Houve melhora em algumas UAPS com salas individualizadas para acolhimento, } \\
\text { respeitando a privacidade do usuário }{ }^{45} \text {. } \\
\text { Desafios } \\
\text { - A ausência de espaço físico adequado para realização do acolhimento diminui sua } \\
\text { potencialidade }{ }^{24,43,44,55,60,61,63} \text {. }\end{array}$ \\
\hline $\begin{array}{l}\text { Tecnologia- } \\
\text { informatica }\end{array}$ & $\begin{array}{l}\text { Avanços } \\
\text { - Facilita o controle e os registros; agiliza o setor de recepção e de prontuários no agendamento } \\
\text { de consultas especializadas (filas virtuais) }{ }^{26,56} \text {. } \\
\text { Desafios } \\
\text { - Aumenta o risco de burocratizar o atendimento, interfere no estabelecimento de uma } \\
\text { relação satisfatória entre o profissional de saúde e o usuário, e coloca em risco a } \\
\text { qualidade do atendimento para uma escuta ampliada }{ }^{56} \text {. } \\
\text { - Permanecem as filas (listas) de espera para especialidade, no plano virtual }{ }^{56} \text {. }\end{array}$ \\
\hline
\end{tabular}

Fonte: Bases de dados Lilacs, Medline e SciELO, no período de 1989 a 2009.

a ausência de mecanismo de comunicação e integração da APS com os demais níveis de complexidade do SUS ${ }^{15,31,43,45,46,53-56}$. Tais resultados demonstram a necessidade de reformular as organizações e os equipamentos de saúde do SUS, criando condições para que estes se aproximem cada vez mais dos seus usuários e comunidades. Nesta direção, a hierarquização rígida do trabalho organizado sob a orientação do eixo de cada nível de complexidade do sistema precisaria ser repensada. Segundo Mendes ${ }^{64}$, quando os níveis de complexidade de um sistema de saúde tornam-se autônomos, há o risco de sua fragmentação, que pode ocorrer entre unidades semelhantes ou em diferentes níveis de complexidade com consequências negativas para o acesso, a eficiência e a equidade. De acordo com Gottems e Pires $^{65}$ a articulação da APS com os demais níveis de complexidade da saúde influenciaria efetivamente na dimensão econômica do SUS, já que poderia interferir na lógica da oferta a partir da demanda, reduzindo iniquidades e impactando as ações de alta complexidade.

Diante disto, surge no cenário nacional a concepção de redes integradas e regionalizadas de atenção à saúde, que devem ser efetivadas pela centralidade das ações nas necessidades dos usuários e na continuidade do cuidado de forma articulada, integrando as diversas unidades e níveis de complexidade do SUS e reconhecendo a com- 
plementaridade e a interdependência de cada unidade e os diferentes níveis de atenção ${ }^{66}$. Cecí1 lio ${ }^{67}$ pontua que para a concretização destas redes, cada serviço deveria se articular em fluxos e circuitos de forma complementar e dialética a partir das necessidades reais dos usuários, buscando agregar os saberes das equipes interdisciplinares e de outros setores para além da saúde.

Neste sentido, o acolhimento deveria ser concebido como uma rede de conversação, conforme assinala Teixeira ${ }^{68}$ como um "acolhimentodiálogo", um autêntico espaço interligado e composto de regiões de conversa, no qual o acolhimento teria o papel de receber, interligar e mover-se por esse espaço, para oferecer aos usuários amplas possibilidades de trânsito no sistema. O "acolhimento-diálogo", desde a perspectiva do desempenho global da rede, pode ser visto como uma espécie de distribuidor e operador desta distribuição, por estar onipresente em todos os pontos da rede. Nesses momentos e espaços de encontros buscar-se-ia o entendimento e a negociação permanente para a formulação da trajetória do usuário pela rede do SUS, porém com sua participação efetiva.

Ademais, vale ressaltar que, quando o profissional de saúde restringe sua atuação a uma pequena parcela do processo terapêutico do usuário, de forma fragmentada, acaba por gerar sua própria desmotivação, ao não conseguir vislumbrar a dimensão global de sua atuação. Para operar neste desafio o Ministério da Saúde ${ }^{69}$ propõe o prontuário unificado e transdisciplinar, que poderia estimular e fortalecer o trabalho em equipe pelo diálogo, pela troca de conhecimentos e por elaborações coletivas em grupo. Além disto, instigaria o profissional para a construção do trabalho centrado no usuário, fortalecendo a sua autonomização ${ }^{43}$.

Outro importante desafio do acolhimento para a operacionalização e a qualificação do SUS, apontado nesta revisão, foi a necessidade de transformar o modelo assistencial ${ }^{25,33,37,43}$. Este, segundo Teixeira et al..$^{70}$, seria uma "combinação de tecnologias acionadas para o enfrentamento de problemas (danos e riscos) e a necessidade de saúde (incluídas as carências e problemas, [...] as oportunidades e projetos de vida pessoal ou de classe de sujeitos sociais)". Deste modo, as transformações necessárias envolveriam a recomposição dos meios de trabalho, a reestruturação das atividades nos serviços de saúde e a redefinição das relações sociais e técnicas sob as quais se realizam o processo de trabalho.

Destaca-se nos resultados dos estudos (Quadro 1), que a partir da adoção da diretriz do aco- lhimento os profissionais se tornaram mais sensíveis às necessidades e à responsabilização com à saúde dos usuários ${ }^{25,33,37,43,59,662}$. No entanto, ainda se observa a manutenção do modelo assistencial centrado no modelo clínico e na organização do processo de trabalho a partir da consulta médi$\mathrm{ca}^{24,36,53}$. Nesta direção, outro desafio confirmado nesta revisão (Quadro 1), foi a pouca valorização dos profissionais pelos saberes, crenças e habilidades dos usuários, cuja tendência leva-os a culpabilizá-los por seus problemas de saúde ${ }^{42,43,57,60,61}$.

Não obstante, o modelo biomédico, adotado historicamente pelo sistema de saúde brasileiro, cuja lógica da assistência à saúde centraliza-se no ato prescritivo de produzir procedimentos (consultas, exames, medicamentos, curativos, vacinas, entre outros), desconsidera os demais determinantes do processo saúde-doença e a subjetividade dos sujeitos ${ }^{71}$. Em decorrência, o processo de trabalho apoiado neste modelo preconiza a concepção biológica e mecanicista do processo saúde-doença, na qual a doença é vista como uma perturbação no funcionamento normal, cabendo aos profissionais de saúde o papel de intervir, física ou quimicamente para corrigir este defeito ou disfunçãa ${ }^{72,73}$. Ora, se a doença é vista como um mau funcionamento do organismo e a equipe de saúde como interventora, resta ao usuário ocupar uma condição passiva e dependente, que marcado por sua condição de adoecimento, percebe-se subjugado e subordinado aos serviços de saúde e aos profissionais disponíveis, se colocando sob sua tutela ${ }^{22,74}$. Por conseguinte, os profissionais de saúde passam a dar assistência por meio de procedimentos técnicos, protocolos, prescrições, ao mesmo tempo em que cobram a obediência dos usuários ${ }^{22,75,76}$.

Neste contexto, vale confrontar os resultados encontrados nos estudos analisados referentes à adoção de protocolos para critérios de riscos, apresentados no Quadro 1. Foi confirmado que o uso destes protocolos garante a priorização do atendimento a partir da identificação de riscos de grupos ou indivíduos que apresentam maior vulnerabilidade de morrer, adoecer, ter comprometida sua qualidade de vida ou limitada sua autonomia funcional. Assim, o seu uso interrompe a perversa ordem de entrada nos serviços de saúde por critérios simplesmente burocráticos e administrativos, como, por exemplo, a distribuição de senhas por ordem de chegada no serviço ${ }^{15,33,36,45,47,61}$.

Em contrapartida, há o risco de manter a avaliação do profissional de saúde somente para a queixa-conduta, centrada nos aspectos clínicos 
dos problemas apresentados pelo usuário e definidos a partir destes protocolos. Ao mesmo tempo, isto pode levar o usuário a exagerar na sua queixa, ao compreender que, o profissional que o recebeu é quem irá decidir (por ele) se deve ou não ser atendido ou por qual profissional será atendido, perpetuando sua insatisfação e limitando sua autonomia ${ }^{49,55,60}$. Para amenizar tais riscos, sugere-se manter a sensibilização, a participação e a compreensão dos profissionais de saúde e dos usuários sobre o uso destes protoco$\operatorname{los}^{15,24,31,47,39}$. Também é necessário, que os profissionais de saúde compreendam a importância de flexibilizar o seu uso para ampliar e qualificar a escuta no ato de acolher ${ }^{42}$.

Ademais, na APS, o modelo biomédico utilizado pelos profissionais de saúde, e muitas vezes demandados pelos usuários ${ }^{15,24,32,57}$, como visto no Quadro 1, deixa pouco espaço para ações educativas, de promoção e de prevenção à saúde. Por conseguinte, há o risco de manter a mesma lógica hegemônica, no embasamento dos programas de saúde, principalmente quando se necessita de ações urgentes para o controle de certas epidemias. Nesta perspectiva, a visão higienista, pontuada apenas como uma questão de hábitos e comportamentos individuais, abstraídos de seu contexto histórico, de suas relações com as condições de vida e de trabalho das populações, poderia levar ao mesmo desfecho, que culpabiliza os usuários por seus problemas de saúde, desconsiderando sua singularidade e submetendo-os novamente à autoridade dos profissionais de saúde $\mathrm{d}^{22,75-77}$.

Ao contrário, ao considerar o estado de saúde em permanente transformação, uma visão ampliada permite romper com a idéia estagnada, fragmentada e disciplinar; o que representará inscrevêla como campo do conhecimento interdisciplinar e como prática social na ordem intersetorial ${ }^{22,78,79}$. Para guiar esta transformação é imprescindível integrar e articular diferentes saberes e práticas, focando na autonomia dos sujeitos e buscando na interdisciplinaridade as potencialidades partilhadas dos diferentes atores, recursos e equipamentos da saúde, e na intersetorialidade novos recur$\operatorname{sos}^{22,64,65,78,79}$. Nesta direção a construção da autonomia e do autocuidado poderia ser alcançada pela mudança da postura tradicional dos profissionais, que coloca os usuários e as comunidades como objeto inerte, que deve obedecer de maneira acrítica e sem restrições as prescrições disciplinares. Ao contrário, dever-se-ia buscar a corresponsabilização, desde a construção do diagnóstico, mapa de vulnerabilidade e riscos até a elaboração do projeto terapêutico $22,39,40,80$.
Trata-se, portanto, de mudanças nas práticas cotidianas dos serviços que considerem os usuários como sujeitos do processo de cuidado, inclusive de escolhas terapêuticas. Para isto, os profissionais precisariam compreender os fatores implicados e as representações presentes nas escolhas terapêuticas e na sua adesão, já que isto poderia estar relacionado à sua satisfação com o serviço e as respostas às necessidades de saúde, ao vínculo construído, como também ao processo de comunicação ${ }^{22,38,39,50,80,81}$.

Assim, faz-se necessário a reconstrução das ações educativas nos serviços de saúde desde a formação do profissional até a produção da Educação Permanente, visando o estabelecimento de uma nova cultura, voltada para os compromissos sociais e para responder as reais demandas da população brasileira ${ }^{73,82}$. Desse modo, aponta-se para um novo desafio apresentado no Quadro 1, relacionado aos poucos mecanismos de acesso para a capacitação dos profissionais de saúde: a Educação Permanente ${ }^{24,31-33,44,55,57,62}$.

Nesta direção, a Organização Pan-americana de Saúde ${ }^{83}$ discute a necessidade de melhorar a atenção prestada pelos profissionais de saúde pelo investimento na sua qualificação e capacitação; enquanto a IX Conferencia Nacional de Saú$\mathrm{de}^{84}$, realizada em 1992, já afirmava que, para implementar e qualificar o SUS, seria indispensável investir na qualificação de seus trabalhadores. Aprovada pelo Conselho Nacional de Saúde, em 2003, destaca-se a Política Nacional de Educação Permanente em Saúde que buscou constituir-se em um eixo transformador do SUS, articulando Educação e Saúde. A Educação Permanente ${ }^{83}$ é entendida como a aprendizagem no cotidiano dos serviços, que utiliza o pressuposto pedagógico da aprendizagem significativa ${ }^{22}$, a partir da problematização do processo de trabalho na busca de soluções coletivas e inovadoras.

Neste caminho será preciso produzir uma nova consciência do sujeito público, que gere inovações para a construção contra-hegemônica de modelos voltados para a produção do cuidado em saúde ${ }^{73,75,82,85}$. Ceccim ${ }^{86}$ alerta que uma política de educação para o SUS precisa envolver não somente os profissionais de saúde que já estão no campo de trabalho, mas também os discentes, docentes, gestores de ensino e de informação científico-tecnológica, procurando produzir uma política intersetorial e de interface.

Outro importante desafio destacado nesta revisão foi a organização do trabalho no âmbito no acolhimento à atenção primária no SUS (Quadro 1), caracterizado pelo excesso de demanda e 
ausência de profissionais, levando à pressão pelo atendimento rápido, à sobrecarga de trabalho e ao sofrimento ${ }^{24,31-33,44,46,55,62}$. Pontuam Scholze et al. ${ }^{39}$ que tais condições podem acarretar riscos a saúde dos profissionais, como por exemplo, a síndrome de Burnout, que afeta geralmente pessoas envolvidas nos cuidados em saúde, quando expostos a excessivos e prolongados estados de tensão no âmbito do trabalho. O sofrimento causado, neste tipo de trabalho, pode levá-los a utilizarem estratégias defensivas, assegurando certa normalidade pela aparente insensibilidade contra o que possa lhe causar sofrimento, postura contrária à preconizada pelos parâmetros da $\mathrm{PNH}$.

Alerta Eugenio Paes Campos ${ }^{87}$ que "o profissional de saúde é um cuidador sob tensão, na medida em que seu objeto de trabalho é [...] alguém que expressa sofrimento e que demanda [...] resultados muitas vezes superiores à possibilidade humana de alcançá-los”. Assim, no cotidiano dos serviços de saúde, os profissionais convivem com um conjunto de angústias, conflitos e obstáculos em sua prática, e por isto, do mesmo modo, demandam cuidados. Para este autor, os profissionais precisariam de espaços para acolhimento, escuta e construção de novas formas de lidar com o estresse do trabalho, por meio da própria dinâmica de funcionamento e do relacionamento da equipe. De acordo com Tesser et al. ${ }^{35}$, "o apoio humano, emocional e institucional para a equipe são necessários, assim como facilitar a construção de clima de equipe, de corresponsabilização e de parceria entre os profissionais; e sua Educação Permanente”.

Já na arena da gestão, Gastão Wagner de Souza Campos ${ }^{88}$ alerta sobre o modelo de cogestão, que busca assegurar o cumprimento primário da organização de saúde, a produção da saúde, ao mesmo tempo em que permite e estimula os profissionais a ampliarem sua capacidade de reflexão e de participação, alcançando maior realização profissional e pessoal. Neste sentido, propõe criar espaços de democracia ampliada, em um tipo de gestão colegiada, fundamentado no conceito de cogestão, no qual todos participam, ou seja, nunca se decide sozinho ou isoladamente, ou pelo outro. Tal proposta vai ao encontro da formulação da $\mathrm{PNH}$, que segundo o Ministério da Saúde ${ }^{89}$ tem como eixo da gestão do trabalho promover ações que garantam a participação dos trabalhadores nos processos de discussão e de decisão, buscando fortalecer e valorizar sua motivação, seu autodesenvolvimento e o crescimento profissional. Assim, o modelo de gestão preconizado pela $\mathrm{PNH}^{90}$ centra-se "no tra- balho em equipe, na construção coletiva (planeja quem executa) e em espaços coletivos que garantem que o poder seja de fato compartilhado, por meio de análises, decisões e avaliações construídas coletivamente".

As práticas cotidianas do acolhimento nos serviços de saúde, no entanto, apontaram contradições, que merecem extrema atenção por parte dos gestores, como apresentadas no Quadro 1: a existência de conflitos e de desarticulação das equipes, a ausência de reuniões ou espaços para a discussão e reflexões das equipes; e a fragmentação do processo de trabalho pela ausência da participação dos profissionais na formulação das propostas dos serviços ${ }^{31,36,43,44,49,55,63}$.

Ademais, em relação à ambiência para o acolhimento, esta deve produzir segurança e privacidade, já que somente assim, poderá realmente potencializar o encontro entre usuário e profissionais no debate em torno da produção de cuidado, de vínculo e de corresponsabilização em saúde. Em contraposição, em alguns estudos anali$\operatorname{sados}^{24,43,44,55,60,61,63}$, evidenciou-se a negligencia deste aspecto na UAPS.

Vale enfatizar, ainda, que o uso de tecnologia informacional, apesar de agilizar o setor de recepção, facilitando o agendamento e os registros de procedimentos realizados ${ }^{26}$, pode burocratizar o acolhimento e interferir negativamente no estabelecimento de uma relação entre o profissional e o usuário satisfatória e primordial para a escuta qualificada, humanizada e resoluta ${ }^{56}$.

\section{Considerações finais}

O acolhimento, ao ampliar o acesso dos usuários ao SUS na APS, quando associado à presença de profissionais capacitados para uma escuta ativa e qualificada às suas demandas, possibilita a autonomia, a cidadania e a corresponsabilização na produção do cuidado à saúde. Além disto, pode contribuir efetivamente para a superação do mito, construído ao longo dos anos, de que as ações de saúde prestadas pelos serviços públicos são de má qualidade e seus profissionais desqualificados, e que os serviços de "ponta" estão no setor privado.

Por outro lado, quando o acolhimento é tomado como um pronto atendimento, triagem a ser realizada na porta do serviço, pode perpetuar a exclusão dos usuários e das comunidades ao SUS, dificultando a adesão ao projeto terapêutico, o vínculo e a corresponsabilização. Além disto, esta prática restrita de acolhimento tem sido 
percebida pelos profissionais de saúde, como mais uma tarefa a ser realizada, entre tantas outras das UAPS, causando sobrecarga de trabalho, cansaço, estresse e conflitos nas equipes, dificultando a qualificação do SUS.

Assim sendo, o acolhimento, em suas diferentes configurações, destaca-se como um processo em construção no SUS, que deve ser capaz de incluir os usuários nos serviços e, ao mesmo tempo, potencializar os profissionais de saúde e gestores na construção de espaços democráticos, éticos e reflexivos para a construção de um novo modelo assistencial, capaz de produzir sujeitos, cuidado e saúde.

\section{Colaboradores}

SM Mitre foi responsável pelo desenho do estudo, pesquisa, redação e revisão do manuscrito. EIG Andrade e RMM Cotta foram responsáveis pelo desenho do estudo, orientação, redação e revisão do manuscrito.

\section{Referências}

1. Organização Pan-Americana da Saúde (OPAS). Declaração de Alma-Ata. In: Conferência Internacional Sobre Cuidados Primários de Saúde, 6-12 set 1978, AlmaAta. [site da Internet] 2008 [acessado 2010 nov 30]. Disponível em: http://www.opas.org.br/promocao/ uploadArq/Alma-Ata.pdf

2. Fleury S. A questão da democracia na saúde. Bases conceituais da reforma sanitária brasileira. In: Fleury S, organizadores. Saúde e Democracia: a luta do CEBES. São Paulo: Lemos Editorial; 1997. p. 25-41.

3. Cordeiro H. Instituto de Medicina Social e a Luta pela Reforma Sanitária: Contribuição à Historia do SUS. Physis 2004; 14(2):343-362.

4. Brasil. Ministério da Saúde (MS). Ministério da Previdência e Assistência Social. Boletim da 8a. Conferencia Nacional de Saúde. Ed. Brasília: DF; 1986.

5. Nascimento PR, Zioni F. O Sistema Único de Saúde como observatório de direitos sociais: uma reflexão a partir das Ciências Sociais. Interface Comun Saúde Educ 2010; 14(32):199-205.

6. Gomes MCPA, Pinheiro R. Acolhimento e vínculo: práticas de integralidade na gestão do cuidado em saúde em grandes centros urbanos. Interface Comun Saúde Educ 2005; 9(17): 287-302.

7. Cotta RMM, Mendes FF, Muniz JN. Descentralização das políticas públicas de saúde: do imaginário ao real. Viçosa: Editora UFV- CEBES; 1998.

8. Andrade EIG, Dias Filho PPS. Padrões de financiamento da saúde do trabalhador: do seguro social ao seguro saúde. In: Lobato LVC, Fleury S, organizadores. Seguridade Social, Cidadania e Saúde. Rio de Janeiro: Cebes; 2010. p.160-172.

9. Faveret Filho P, Oliveira PJ. A universalização excludente: reflexões sobre as tendências do sistema de saúde. Dados 1999; 33(2):257-283.

10. Silva GR. O SUS e a crise atual do setor público de saúde. Saude Soc 1995; 4(2):15-21. 
11. Rollo A, Oliveira RC. É possível construir novas práticas assistenciais no hospital público? In: Merhy EE, Onocko R. organizadores. O agir em saúde: um desafio para o público. São Paulo, Buenos Aires: Editora Hucitec, Lugar Editorial; 1997. p. 321-352.

12. Cecílio LCO. Modelos tecno-assistenciais em saúde: da pirâmide ao circulo, uma possibilidade a ser explorada. Cad Saude Publica 1997; 13(3):469-475.

13. Merhy EE. A perda da dimensão cuidadora na produção de cuidado: uma discussão do modelo assistencial e da intervenção no seu modo de trabalhar a assistência. In: Campo CR, Malta DC, Reis AT, Santos AF, Merhy EE, organizadores. O sistema Único de Saúde de Belo Horizonte: reescrevendo o público. São Paulo: Xamã; 1998. p.103-120.

14. Merhy EE. O SUS e um dos seus Dilemas: mudar a gestão e a lógica do processo de trabalho em saúde: um ensaio sobre a micropolítica do trabalho vivo. In: Fleury S. organizadores. Saúde e Democracia: a luta do CEBES. São Paulo: Lemos Editorial; 1997. p. 125-141.

15. Franco TB, Bueno WS, Merhy EE. O acolhimento e os processos de trabalho em saúde: o caso de Betim (MG). Cad Saude Publica 1999; 15(2):345-353.

16. Malta DC, Ferreira LM, Reis AT, Merhy EE. Acolhimento: uma reconfiguração do processo de trabalho em saúde usuário-centrada. In: Campo CR, Malta DC, Reis AT, Santos AF, Merhy É, organizadores. O sistema Único de Saúde de Belo Horizonte: reescrevendo o público. São Paulo: Xamã; 1998. p 121-142.

17. Benevides R, Passos E. Humanização na saúde: um novo modismo? Interface Comun Saúde Educ 2005; 9(17):398-406.

18. Brasil. Ministério da Saúde (MS). Conselho Nacional de Saúde. 11 ${ }^{a}$. Conferencia Nacional de Saúde. O Brasil falando como quer ser tratado: efetivando o SUS - acesso, qualidade e humanização na atenção à saúde com controle social. [Relatório final]. Brasília: MS; 2001.

19. Brasil. Ministério da Saúde (MS). 12a. Conferencia Nacional de Saúde. Conferência Sergio Arouca. Brasília, 7 a 11 de dezembro de 2003. Relatório final/ Ministério da Saúde, Conselho Nacional de Saúde. Brasília: MS; 2004.

20. Santos-Filho SB, Barros MEB, Gomes RS. A Política Nacional de Humanização como política que se faz no processo de trabalho em saúde. Interface Comun Saúde Educ 2009; 13(Supl.1):603-613.

21. Brasil. Ministério da Saúde (MS). Secretaria de Atenção à Saúde. Núcleo Técnico da Política Nacional de Humanização. Acolhimento nas práticas de produção de saúde. Ministério da Saúde, Secretaria de Atenção à Saúde, Núcleo Técnico da Política Nacional de Humanização. 2a ed. Brasília: MS; 2006.

22. Mitre SM. Avanços e Desafios do acolhimento na reabilitação: um estudo dos Centros de Referência em Reabilitação da Rede do Sistema Único de Saúde de Belo Horizonte, MG [dissertação]. Belo Horizonte (MG): Universidade Federal De Minas Gerais; 2011.

23. Bardin L. Análise de Conteúdo. Lisboa: Edições 70; 2008.

24. Leite JCA, Maia CCA, SENA RR. Acolhimento: perspectiva de reorganização da assistência de enfermagem. Rev. Bras. Enferm 1999; 52(2):161-168.
25. Camelo SHH, Angerami ELS, Silva EM, Mishima SM. Acolhimento à clientela: estudo em unidades básicas de saúde no município de Ribeirão Preto. Rev. Latino-Am. Enfermagem 2000; 8(4):30-37.

26. Moresco PM, Souza IG, Pimentel RM. Avaliação da informação e do acolhimento solidário na Unidade de Saúde Vila Clarice. Saúde para Debate 2000; 19:40-43.

27. Benevides R, Passos E. A humanização como dimensão pública das políticas de Saúde. Cien Saude Colet 2005; 10(3):561-571.

28. Minayo MCS. Estrutura e sujeito, determinismo e protagonismo histórico: uma reflexão sobre a práxis da saúde coletiva. Cien Saude Colet 2001; 6(1):7-19.

29. Santos-Filho SB. Avaliação e humanização em saúde: aproximações metodológicas. Ijuí: Ed. Unijuí; 2009.

30. Ayres JRC. Sujeito, intersubjetividade e práticas de saúde. Cien Saude Colet 2001; 6(1):63-72.

31. Sena-Chompré R, Leite JCA, Maia CCA, Gonzaga RL, Santos FCO. Acolhimento como mecanismo de implementação do cuidado de enfermagem. Cogitare Enferm 2000; 5(2):51-57.

32. Schimith MD, Lima MADS. Acolhimento e vínculo em uma equipe do Programa Saúde da Família. Cad Saude Publica 2004; 20(6):1487-1494.

33. Silveira MFA, Felix LG, Araújo DV, Silva LC. Acolhimento no Programa Saúde da Família: um caminho para a humanização da atenção à saúde. Cogitare Enferm 2004; 9(1):71-78.

34. Cunha GT. A construção da clínica ampliada na atenção básica. 3a ed. São Paulo: Hucitec; 2010.

35. Tesser DC, Poli Neto P, Campos GWS. Acolhimento e (dês) medicalização social: um desafio para as equipes de saúde da família. Cien Saude Colet 2010; 15(Supl. 3):3615-3624.

36. Takemoto MLS, Silva EM. Acolhimento e transformações no processo de trabalho de enfermagem em unidades básicas de Campinas, São Paulo, Brasil. Cad Saude Publica 2007; 23(2):331-340.

37. Ramos DD, Lima MADS. Acesso e acolhimento aos usuários em uma unidade de saúde de Porto Alegre, Rio grande do Sul. Cad Saude Publica 2003; 19(1):27-34.

38. Cotta RMM, Gomes AP, Maiua TM, Magalhães KA, Marques ES, Siqueira-Batista R. Pobreza, injustiça, e desigualdade social: repensando a formação de profissionais de saúde. Rev. bras. educ. med 2007; 31(2):278-286.

39. Scholze AS, Duarte Junior CF, Silva FY. Trabalho em saúde e a implantação do acolhimento na atenção primária à saúde: afeto, empatia ou alteridade? Interface Comun Saúde Educ 2009; 13(31):303-314.

40. Ayres JRC. O cuidado, os modos de ser (do) humano e as práticas de saúde. Saude Soc 2004; 13(3):16-29.

41. Brasil. Ministério da Saúde (MS). Secretaria de Atenção à Saúde. Núcleo Técnico da Política Nacional de Humanização. Humaniza SUS: Documento base para gestores e trabalhadores do SUS. $4^{\circ}$ ed. Brasília: Editora do MS; 2008.

42. Cavalcante Filho JB, Vasconcelos SEM, Ceccim RB, Gomes LB. Acolhimento coletivo: um desafio instituinte de novas formas de produzir o cuidado. Interface Comun Saúde Educ 2009; 13(31):315-328. 
43. Coelho MO, Jorge MSB. Tecnologia das relações como dispositivo do atendimento humanizado na atenção básica na perspectiva do acesso, do acolhimento e do vínculo. Cien Saude Colet 2009; 14

44. (Supl. 1):1523-1531.

Nery SR, Nunes EFPA, Carvalho BG, Melchior R, Baduy RS, Lima JVC. Acolhimento no cotidiano dos auxiliares de enfermagem nas Unidades de Saúde da Família, Londrina (PR). Cien Saude Colet 2009; 14(Supl.

45. 1):1411-1419.

Esmeraldo GROV, Oliveira LC, Souza KMM, Araújo MAM, Esmeraldo-Filho CE, Viana EMN. Análise do acolhimento na estratégia de saúde da família sob a perspectiva do usuário. Rev APS 2009; 12(2):

46. $112-130$

Pinafo E, Lima JVC, Baduy RS. Acolhimento: concepção dos auxiliares de enfermagem e percepção de usuários em uma unidade de saúde da família.

47. Espaç Saúde 2008; 9(2):17-25.

Zaicaner R, Basso ACPL, Lima HG, Buchala MZ, Pereira OF. Análise da implantação do protocolo para incorporação de pacientes de grupos prioritários na atenção básica. Saude para Debate 2008; 42:90-

48. 101.

Silveira MGG, Baldacci ER. Humanização e autogestão em uma unidade básica de saúde. Pediatria

49. 2006; 28(4):226-233.

Donabedian A. Garantí y monitoria de la calidad de la atención médica. México: Instituto Nacional de Salud

50. Pública; 1999.

Tavassos C, Martins M. Uma revisão sobre o conceito de acesso e utilização de serviços de saúde. Cad. Saúde

51. Pública 2004; 20(Supl. 2):190-198.

Malta DC, Merhy EE. A Avaliação do projeto vida e do acolhimento no Sistema Único de Saúde de Belo Hori-

52. zonte. Rem: Rev. Esc. Minas 2004; 8(2):259-267. Brasil. Ministério da Saúde (MS). O SUS de A a Z: garantindo saúde nos municípios. Ministério da Saúde, Conselho Nacional de Secretários Municipais de Saúde.

53. Brasília: MS; 2005.

Souza CF, Vilar RLA, Rocha NSPD, Uchoa AC, Rocha PM. Acesso e acolhimento na atenção básica: uma análise da percepção dos usuários e profissionais de saúde.

54. Cad Saude Publica 2008; 24(Supl. 1):100-110.

Santos AM, Assis MMA, Rodrigues AAO, Nascimento MAAA, Jorge Bessa MA. Linhas de tensões no processo de acolhimento das equipes de saúde bucal do Programa de Saúde da Família: o caso de Alagoinhas, Bahia,

55. Brasil. Cad Saude Publica 2007; 23(1):75-85. Fracolli LA, Zoboli ELCP. Descrição e análise do acolhimento: uma contribuição para o programa de saúde da

56. família. Rev Esc Enferm USP 2008; 38(2):143-151. Maciel-Lima SM. Acolhimento solidário ou atropelamento? A qualidade na relação profissional de saúde e paciente face à tecnologia informacional. Cad Saude Pu-
57. blica 2004; 20(2):502-511.

Oliveira A, Neto Silva JC, Machado MLT, Souza MBB, Feliciano AB, Ogata MN. A comunicação no contexto do acolhimento em uma unidade de saúde da familiar de São Carlos. SP. Interface Comun

58. Saúde Educ 2008; 12(27):749-762.

Lima MADS, Ramos DD, Rosa RB, Nauderer TM, Davis R. Acesso e acolhimento em unidades de

59. saúde na visão dos usuários. Acta paul. enferm 2007; 20(1):12-17.

Monteiro MM, Figueiredo VP, Machado MFAS. Formação do vínculo na implantação do Programa Saúde

60. da Familia numa Unidade Básica de Saúde. Rev Esc Enferm USP 2009; 43(2):358-364.

Matumoto S, Mishima SM, Fortuna CM, Pereira MJB, Almeida MCP. Preparando a relação de atendimento:

61. ferramenta para o acolhimento em unidades de saúde. Rev. Latino-Am. Enfermagem 2009; 17(6):1001-1008. Pina JC, Mello DF, Mishima SM, Lunardelo SR. Contribuições da estratégia Atenção Integrada às Doenças Pre-

62. valentes na Infância ao acolhimento de crianças menores de cinco anos. Acta paul. enferm 2009; 22(2):142-48. Ayres RCV, Pereira SPOE, Ávila SMN, Valentim W. Aco-

63. lhimento no PSF: humanização e solidariedade. Mundo Saúde 2006; 30(2):306-311.

Freire LAM, Storino LP, Horta NC, Magalhães RP, Lima T. O acolhimento sob a ótica de profissionais da equipe

64. de saúde da família. Rem: Rev. Esc. Minas 2008; 12(2):271-277.

Mendes EV. Os Sistemas de Serviços de Saúde: o que os gestores deveriam saber sobre essas organizações com-

65. plexas. Fortaleza (CE): Escola de Saúde Pública do Ceará; 2006.

Gottems LBD, Pires MRGM. Para além da atenção básica: reorganização do SUS por meio da interse-

66. ção do setor político com o econômico. Saude Soc 2009; 18(2):189-198.

Brasil. Ministério da Saúde (MS). Conselho Nacional de Secretários de Saúde. Sistema Único de Saúde: avanços e desafios. Conselho Nacional de Secretários de Saúde. Ministério da Saúde (MS). Conselho

67. Nacional de Secretários de Saúde. Brasília: CONASS; 2006.

Cecílio LCO. As necessidades de saúde como conceito estruturante. In: Pinheiro R, Mattos RA, organizadores. Os sentidos da integralidade na atenção

68. e no cuidado à saúde. Rio de Janeiro: CEPESC/UERJ, IMS, Abrasco; 2006. p.113-127.

Teixeira RR. O acolhimento num serviço de saúde entendido como uma prática de conversação. In: Pinheiro R, Mattos RA, organizadores. Construção da Integralidade: cotidiano, saberes e práticas em 
69. saúde. Rio de Janeiro: IMS/UERJ/CEPESC/Abrasco; 2007. p. 89-112.

Brasil. Ministério da Saúde (MS). Secretaria-Executiva. Núcleo Técnico da Política Nacional de Humanização. HumanizaSUS: prontuário transdisciplinar e projeto terapêutico. Ministério da Saúde,

70. Secretaria Executiva, Núcleo Técnico da Política Nacional de Humanização. Brasília: MS; 2004.

Teixeira CF, Paim JS, Vilas Bôas AL. SUS: modelos

71. assistenciais e vigilância em saúde. Inform Epidemiol SUS 1998; 7(2):7-28.

Matumoto S, Fortuna CM, Mishima SM, Pereira MJB, Domingos NAM. Supervisão de equipes no programa de saúde da família: reflexões acerca do desafio da pro-

72. dução de cuidados. Interface Comun Saúde Educ 2005; 9(16):9-24.

Capra F. O ponto de mutação: a ciência, a sociedade

73. e a cultura emergente. Trad.: Álvaro Cabral. São Paulo: Cultrix; 2006.

Mitre S M, Siqueira-Batista R, Girardi-de-Mendonça J M, Morais-Pinto N M, Meirelles C A B, Pinto-Porto C, Moreira T, Hoffmann LMA. Metodologias ativas de ensino-aprendizagem na formação dos profissionais de

74. saúde: debates atuais. Cien Saude Colet. 2008; 13(Supl. 2):2133-2144.

75. Herzog R. A percepção de si com sujeito-da-doença. Physis 1991; 1(2):143-155.

Cotta RMM, Schott M, Azeredo CM, Franceschini SC, Priore SE, Dias G. Organização do trabalho e perfil dos profissionais de Programa da Família:

76. um desafio na reestruturação da atenção básica em saúde. Epidemiol. Serv Saúde 2006; 15(3):7-18.

Lacerda A, Valla V. Homeopatia e apoio social. In: Pinheiro R, Mattos RA organizadores. Construção da Integralidade: cotidiano, saberes e práticas em saú-

77. de. Rio de Janeiro: CEPES/ UERJ, IMS, Abrasco; 2007. p 171-198.

78. Valla VV, Stotz EN. Educação, saúde e cidadania. Petrópolis; Rio de Janeiro: Vozes; 1994.

79. Mendes EV. Uma agenda para saúde. São Paulo: Hucitec; 2006.

Santos JL, Wetphal M F. Práticas emergentes de um

80. novo paradigma de saúde: o papel da universidade. Estud Av 1999; 13(35):71-88.

Amaral MA, Campos GWS. A clínica ampliada e compartilhada, a gestão democrática e redes de atenção como referenciais teórico-operacionais

81. para a reforma do hospital. Cien Saude Colet 2007; 12(4):849-859.

Oliveira MC. Os modelos de cuidado como eixo de estruturação de atividades interdisciplinares e mul-
82. tiprofissionais em Saúde. Rev. bras. educ. med 2008; 32(3):347-355.

Campos EF, Pierantoni CR, Viana ALD, Faria RMB, Haddad AE. Os desafios atuais para a educação permanente no SUS. In: Ministério da Saúde, Secretaria de Gestão do Trabalho e da Educação na

83. Saúde. Cadernos de RH Saúde. Recursos humanos em saúde. Brasília: MS; 2006. p. 39-43.

OPAS. Organização Pan-Americana da Saúde. Avalia-

84. ção de tendências e prioridades sobre recursos humanos de saúde. Brasília: OPAS; 2002.

85. Brasil. Ministério da Saúde (MS). Relatório Final da 9a. Conferencia Nacional de Saúde. Brasília: MS; 1993.

Gomes KO, Cotta RMM, Cherchiglia ML, Mitre SM, Batista. A práxis do agente comunitário de saúde no con-

86. texto do programa de saúde da família: reflexões estratégicas. Saude Soc 2009; 18(4):744-755.

Ceccim RB. Educação Permanente em saúde: descentrali-

87. zação e disseminação de capacidade pedagógica na saúde. Cienc Saude Colet 2005; 10(4):973-986.

Campos EP. Quem cuida dos cuidadores? Implantação

88. de um projeto voltado para os profissionais de um hospital de ensino. RAS 2008; 10(4):151-158.

Campos GWS. O anti- Taylor: sobre a invenção de um método para co-governar instituições de saúde pro-

89. duzindo liberdade e compromisso. Cad Saude Publica 1998; 14(4):863-870.

Brasil. Ministério da Saúde (MS). Secretaria Executiva. Núcleo Técnico da Política Nacional de Humanização. Humaniza SUS - Política Nacional de

90. Humanização: documento base para gestores e trabalhadores do SUS. Brasília: MS; 2004.

Brasil. Ministério da Saúde (MS). Secretaria de Atenção à Saúde. Política Nacional de Humanização da Atenção e Gestão do SUS. Gestão participativa e cogestão do SUS. Brasília: MS; 2009.
Artigo apresentado em 28/04/2011

Aprovado em 17/06/2011

Versão final apresentada em 05/07/2011 